ORIGINAL ARTICLE

\title{
Challenges of PMTCT Service Utilization in Amhara Region: A Comparative Cross-sectional Study
}

\author{
Berhanu Elfu Feleke', Belaynew Wasie ${ }^{2 *}$
}

\footnotetext{
OPEN ACCESS

Citation: Berhanu Elfu Feleke, Belaynew Wasie. Challenges of PMTCT Service Utilization in Amhara Region: A Comparative Study.Ethiop J Sci.2018;28(6):779.

doi:http://dx.doi.org/10.4314/ejhs.v28i6.13

Received: December 19, 2017

Accepted: May 11, 2018

Published: November 1, 2018

Copyright: (C) 2018 Berhanu Elfa Feleke, et al. This is an open access article distributed under the terms of the Creative Commons Attribution License, which permits unrestricted use, distribution, and reproduction in any medium, provided the original author and source are credited.

Funding: Cost of the research work was supported by Bahir Dar university Competing Interests: The authors declare that this manuscript was approved by all authors in its form and that no competing interest exists. Affiliation and Correspondence:

${ }^{1}$ Department of Epidemiology and

Biostatistics, University of Bahir Dar, Bahir Dar, Ethiopia

${ }^{2}$ Department of Epidemiology and Biostatistics, University of Bahir Dar, Bahir Dar, Ethiopian

*Email: bewassie@gmail.com
}

\author{
ABSTRACT
}

BACKGROUND: Globally, 370,000 children became newly infected with $H I V$ and an estimated 42,000-60,000 pregnant woman died because of HIV annually. Pediatric HIV infection in 90\% of the cases was acquired from their mothers.

METHODS: Comparative cross-sectional study design was implemented. Epi-info software was used to calculate the sample size, and the estimated sample size was 2667 pregnant women. Data were collected using exit interview technique. Descriptive statistical techniques were used to identify the prevalence of PMTCT service utilization and binary logistic regression was used to identify the determinants of PMTCT service utilization.

RESULTS: A total of 2615 respondents were included for a response rate of $98.05 \%$. Only $61.3 \%$ of the pregnant women attending $A N C$ in the health facility were utilizing PMTCT services [95\% CI: $59 \%-63 \%]$. Around 3.22\% of tested pregnant women did not receive their result. PMTCT service utilization was affected by knowledge of PMTCT [AOR 1.87, $95 \%$ CI: 1.53-2.28], residence [AOR 0.67, $95 \%$ CI: 0.51-0.89], internal referral system [AOR 3.06, 95\% CI: 2.51-3.72], health professional client interaction [AOR 2.18, 95\% CI: 1.75-2.76], fear [AOR 1.23, 95\% CI: 1.03-1.47], long waiting time [AOR 0.74, 95\% CI: 0.58-0.94], number of ANC visit [AOR 1.79, 95\% CI: 1.4-2.29], gravidity [AOR 0.55, 95\% CI: 0.46-0.66], educational status [AOR 1.43, 95\% CI: 1.04-1.96] and involvement in PMTCT services [AOR 2.57, 95\% CI: $1.51-4.4]$.

CONCLUSION: PMTCT service utilization was low in the study area. The presence of internal referral system significantly increases PMTCT service utilization.

KEYWORDS: PMTCT, Service, Utilization, Determinants, Amhara Region

\section{INTRODUCTION}

Mother to child transmission of HIV/AIDS was among mechanism of infecting the new born. Without any intervention, one-third of the child born from HIV infected women will receive the virus. This transmission may occur during pregnancy, labor and delivery or breast-feeding (1). 
Globally, 370,000 children became newly infected with HIV and an estimated 42,00060,000 pregnant woman died because of HIV per year (2). Sub-Saharan Africa constitute $68 \%$ of the world HIV infected population (1). In Ethiopia, $2.1 \%$ of the population was infected with HIV/AIDS. The prevalence of HIV among ANC client was $2.03 \%(3,4)$. Mother-to-child transmission of HIV contribute to $90 \%$ pediatric HIV infection $(5,6)$.

In Amhara Region, the prevalence of HIV/AIDS among women of reproductive age group was $2.2 \%$. Counseling and testing for HIV/AIDS was given only for $10.3 \%$ of pregnant women $(6,7)$. PMTCT service utilization was affected by stigma and discrimination, educational status, income, delayed PMTCT visit, unknown partner sero-status, the number of ANC visit already accomplished, delayed counseling, fear, interaction with health personnel, bad obstetric history, gravidity, knowledge regarding PMTCT, involvement in PMTCT service, referral procedures (6-18).

In Ethiopia, prevention of mother-to-child transmission of HIV service coverage increased by $5.16 \%$ annually, but the utilization of PMTCT service was less than $11 \%$. Every working day, 326 mothers present themselves to ANC missed for HIV testing (19). With all PMTCT service interventions, mother-to-child transmission of HIV can be decreased as low as $1 \% \quad(20,21)$. However around $90 \%$ of pregnant women were not utilizing PMTCT services.

This study will examine the reason why pregnant women fail to use PMTCT services. Decision maker can get valuable evidence to plan effective PMTCT service interventions. The objectives of this study was to estimate the prevalence and determinants of PMTCT service utilization among ANC attendants in resource limited settings.

\section{METHODS AND MATERIALS}

Institution-based comparative cross-sectional study was conducted in the urban and rural setting of Amhara Region. The study was conducted in Bahir Dar City (urban) and Achefer woreda (rural). Bahir Dar City is the capital of Amhara
Regional State located at the geographical coordinates of $11^{\circ} 38^{\prime}$ north latitudes and $37^{\circ} 15^{\prime}$ east longitudes. Achefer Woreda was divided into the Semen and Debube woredas. Semen Achefer Woreda contains 26 kebeles and 7 health centers. Debube Achefer Woreda contains 7 health centers and 20 Kebeles.

The sample size was calculated using Epiinfo software with the assumption of $95 \%$ Confidence interval, rural to an urban ratio of 2 , a power of $85 \%, 50 \%$ proportion of PMTCT service utilization in the rural area, an odds ratio of 1.5 and design effect of 2 giving an estimated 2667 pregnant women.

First, we selected the health centers using simple random sampling technique. Bahir Dar City contains 10 health centers and 5 health centers were selected, repectively, by lottery method. From Semen and Debube Achefer, 7 health centers were selected from the total of 14 health centers using simple random sampling technique. Women coming for ANC visit were selected for exit interview using systematic random sampling technique.

Pregnant women who presented themselves to health institution for ANC visit were included. Pregnant women who were unable to communicate properly were excluded.

The data were collected using exit interview. Clients were interviewed by using structured questionnaire. The data were collected by 15 nurses and supervised by 5 health officers. To ensure the quality of this work, the questionnaire was prepared in English and then translated into Amharic (local language) and then back translated to English to check its consistency. Training was given to the data collectors and supervisors. Pretest was conducted on $5 \%$ of the samples and necessary corrections were made on the questionnaire. The whole data collection process was closely supervised. Data were collected from January 17/2015- April 28/2015.

Data were entered into to the computer using Epi-info software version 7 and transferred to SPSS version 20 for analysis. Descriptive statistical techniques were used to identify the prevalence of PMTCT service utilization. Binary logistic regression with AOR (adjusted odds ratio) 
and their 95\% CI were used to identify the predictors of PMTCT service utilization.

Ethical clearance was obtained from Amhara National Regional State Health Bureau Ethical Review Committee and Bahir Dar University CMHS (college of medicine and health science) Ethical Committee. Official permission was obtained from each health institution. The purpose of the study was explained for all the study participants. Each study participant's right to withdraw from the study at any point was respected. The confidentiality of the data was kept at all stages. Written informed consent was obtained from each participant.

\section{RESULTS}

A total of 2615 pregnant women were included in the study giving a response rate of $98.05 \%$. The average age of the respondents was 24.31 years [SD (standard deviation) 6.83 years]. During the time of interview, $73.8 \%$ of the respondents had only one antenatal care visit (Table 1).

Table 1: Population profile of the study participants $(n=2615)$.

\begin{tabular}{llll}
\hline Variable & & Frequency & Percentage \\
\hline ANC visit & 1 & 1931 & 73.8 \\
& 2 & 507 & 19.4 \\
Residence & 3 & 66 & 2.5 \\
Educational status & 4 & 111 & 4.2 \\
& Rural & 1732 & 66.2 \\
& Urban & 883 & 33.8 \\
Average monthly income & Unable to read and write & 266 & 10.2 \\
& Elementary education & 1709 & 65.4 \\
& Secondary education & 171 & 6.5 \\
& Higher education & 469 & 17.9 \\
Partner occupation & $<500$ birr & 1801 & 69.1 \\
& 500-1000 birr & 316 & 12.1 \\
& 1001-1500 birr & 302 & 11.5 \\
& 1501-2000 birr & 103 & 3.9 \\
& $>2000$ birr & 172 & 6.6 \\
Marital status & Farmer & 374 & 14.3 \\
& Merchant & 1172 & 44.8 \\
& Government employ & 953 & 36.4 \\
& NGO employ & 116 & 4.4 \\
Occupation & Single & 2 & 0.1 \\
& Married & 2603 & 99.5 \\
& Divorced & 7 & 0.3 \\
& Widowed & 3 & 0.1 \\
& House wife & 1912 & 73.1 \\
& Government employ & 400 & 15.3 \\
& Merchant & 99 & 3.8 \\
& NGO employ & 66 & 2.5 \\
& Farmer & 116 & 4.4 \\
& Others & 22 & 0.8 \\
\hline
\end{tabular}

Utilization of PMTCT service: The overall prevalence of PMTCT service utilization among ANC attendants was $61.3 \%$ [95\% CI: $59 \%-63$ $\%$; $38.7 \%$ of ANC attending pregnant women were not utilizing PMTCT services. Around
$3.22 \%$ of tested pregnant women did not receive their result. Only $30.11 \%$ of the mothers had disclosed their result to their partner. Around 50\% of the pregnant women did not have knowledge regarding PMTCT. More than half $(55.5 \%)$ of the 
pregnant mothers did not start ANC at proper time. Around $31.3 \%$ of the pregnant women did not remember their LMP (last menstrual period). After adjusting for residence, knowledge of PMTCT, health facility internal referral system, waiting time, interaction with health professionals, knowledge of partner status, partner involvement, history of bad obstetric outcome, fear, number of ANC visit, educational status, occupation, average monthly income, gravidity and age and PMTCT service utilization were associated with knowledge of partner sero status, residence, health facility internal referral system, interaction with health professional, waiting time, fear, number of ANC visit, gravidity, educational status, involvement in PMTCT service.

The odds of PMTCT service utilization among PMTCT knowledgeable women were 1.87 times higher than non-knowledgeable pregnant women [AOR 1.87, $95 \%$ CI: 1.53-2.28]. The odds of PMTCT service utilization was 33\% higher among rural pregnant women [AOR 0.67, $95 \%$ CI: 0.51-0.89]. The presence of internal referral system within the health facility increases utilization of PMTCT services by 3.06 folds
[AOR 3.06, 95\% CI: 2.51-3.72]. In the presence of good health professional client interaction, the odds of PMTCT service utilization increased by 2.18 folds [AOR 2.18, 95\% CI: 1.75-2.76]. Around $41 \%$ of pregnant women fear about HIV/AIDS. Fear increases PMTCT service utilization by 1.23 times higher [AOR 1.23, 95\% CI: 1.03-1.47]. Long waiting hour inside health facility decreases the utilization of PMTCT service by $26 \%$ [AOR 0.74, 95\% CI: 0.58-0.94]. Pregnant women who had more than one ANC visit were 1.79 times more likely to use PMTCT services as compared to women with only one ANC visit [AOR 1.79, 95\% CI: 1.4-2.29]. The odds of PMTCT service utilization among multigravid women were $45 \%$ higher than primi-gravid women [AOR $0.55,95 \%$ CI: $0.46-0.66$ ]. The odds of PMTCT service utilization among women having diploma and above educational status was 1.43 times. [AOR 1.43, 95\% CI: 1.04 - 1.96]. Only $4.44 \%$ of women were involved in PMTCT service intervention, involving women in PMTCT service increases the utilization of PMTCT service by 2.57 times [AOR 2.57, 95\% CI: 1.51 4.4] (Table 2).

Table 2: Predictors of PMTCT service utilization in rural and urban area's $(\mathrm{n}=2615)$.

\begin{tabular}{|c|c|c|c|c|c|c|}
\hline \multirow[t]{2}{*}{ Variable } & & \multicolumn{2}{|c|}{ Utilization } & \multirow[t]{2}{*}{ COR $[95 \% C I]$} & \multirow[t]{2}{*}{$A O R[95 \% C I]$} & \multirow[t]{2}{*}{$p$-value } \\
\hline & & Yes & No & & & \\
\hline \multirow{2}{*}{$\begin{array}{l}\text { Knowledge of partner } \\
\text { Sero status }\end{array}$} & Yes & 465 & 227 & $1.42[1.17-1.71]$ & $1.87[1.53-2.28]$ & $<0.01$ \\
\hline & no & 1137 & 786 & & & \\
\hline \multirow[t]{2}{*}{ Residence } & Urban & 601 & 282 & $1.56[1.31-1.85]$ & 0.67 [ $0.51-0.89]$ & $<0.01$ \\
\hline & Rural & 1001 & 731 & & & \\
\hline \multirow[t]{2}{*}{ Internal referral system } & Available & 1291 & 591 & $2.96[2.48-3.55]$ & $3.06[2.51-3.72]$ & $<0.01$ \\
\hline & Not & 311 & 422 & & & \\
\hline \multirow{4}{*}{$\begin{array}{l}\text { Interaction with health } \\
\text { worker } \\
\text { Waiting time }\end{array}$} & Good & 1210 & 662 & $1.64[1.37-1.95]$ & $2.18[1.72-2.76]$ & $<0.01$ \\
\hline & Bad & 392 & 351 & & & \\
\hline & Long & 984 & 616 & $1.03[0.87-1.21]$ & $0.74[0.58-0.94]$ & 0.02 \\
\hline & Not & 618 & 397 & & & \\
\hline \multirow[t]{2}{*}{ Fear } & Yes & 663 & 410 & $1.04[0.88-1.22]$ & $1.23[1.03-1.47]$ & 0.02 \\
\hline & No & 939 & 603 & & & \\
\hline \multirow[t]{2}{*}{ Number of ANC } & More than 1 & 504 & 180 & $2.12[1.74-2.59]$ & $1.79[1.4-2.29]$ & $<0.01$ \\
\hline & 1 & 1098 & 833 & & & \\
\hline \multirow[t]{2}{*}{ Gravidity } & Primi-gravid & 530 & 496 & $0.52[0.44-0.61]$ & $0.55[0.46-0.66]$ & $<0.01$ \\
\hline & Multi-gravid & 1072 & 517 & & & \\
\hline \multirow[t]{2}{*}{ Educational status } & Diploma and above & 244 & 84 & $1.99[1.52-2.6]$ & $1.43[1.04-1.96]$ & 0.02 \\
\hline & Below diploma & 1358 & 929 & & & \\
\hline \multirow{2}{*}{$\begin{array}{l}\text { Involvement in PMTCT } \\
\text { service }\end{array}$} & Yes & 97 & 19 & $3.37[2-5.73]$ & $2.57[1.51-4.4]$ & $<0.01$ \\
\hline & No & 1505 & 994 & & & \\
\hline
\end{tabular}

DOI: http://dx.doi.org/10.4314/ejhs.v28i6.13 
PMTCT service utilization in rural area: Utilization of PMTCT service among ANC attendants in rural area was $57.8 \%$ [95 \% CI: 55 $\%-60 \%$ ]. PMTCT service utilization in rural area was affected by knowledge of partner sero-status, starting ANC visit at proper time, the presence of internal referral system in the health facility, good interaction with health care personnel, waiting hour, number of ANC visit, gravidity and occupation of the women (Table 3 ).

Table 3: Determinants of PMTCT service utilization in rural area $(n=1732)$

\begin{tabular}{|c|c|c|c|c|c|c|}
\hline \multirow[t]{2}{*}{ Variable } & & \multicolumn{2}{|c|}{ Utilization } & \multirow[t]{2}{*}{ COR $[95 \% \mathrm{CI}]$} & \multirow[t]{2}{*}{ AOR $[95 \% \mathrm{CI}]$} & \multirow[t]{2}{*}{ p-value } \\
\hline & & Yes & No & & & \\
\hline \multirow[t]{2}{*}{ Knowledge of partner Sero status } & Yes & 350 & 180 & $1.65[1.32-2.05]$ & $2.58[2.01-3.3]$ & $<0.01$ \\
\hline & No & 651 & 551 & & & \\
\hline \multirow[t]{2}{*}{ Fist ANC date } & Proper & 250 & 224 & $0.75[0.61-0.94]$ & 1.4 [1.07-1.84] & 0.02 \\
\hline & Not proper & 751 & 507 & & & \\
\hline \multirow[t]{2}{*}{ Internal referral system } & Available & 729 & 346 & $2.98[2.43-3.67]$ & 4.14 [3.29-5.22] & $<0.01$ \\
\hline & Not & 272 & 385 & & & \\
\hline \multirow[t]{2}{*}{ Interaction with health worker } & Good & 616 & 382 & $1.46[1.2-1.78]$ & $2.41[1.87-3.12]$ & $<0.01$ \\
\hline & $\mathrm{Bad}$ & 385 & 349 & & & \\
\hline \multirow[t]{2}{*}{ Waiting time } & Long & 390 & 336 & $0.75[0.62-0.91]$ & $0.68[0.52-0.88]$ & 0.02 \\
\hline & Not & 611 & 395 & & & \\
\hline \multirow[t]{2}{*}{ Number of ANC } & More than 1 & 139 & 30 & $3.77[2.47-5.78]$ & 4.48 [2.81-7.43] & $<0.01$ \\
\hline & Only 1 & 862 & 701 & & & \\
\hline \multirow[t]{2}{*}{ Gravidity } & Primi-gravid & 339 & 344 & $0.58[0.47-0.7]$ & $0.43[0.33-0.56]$ & $<0.01$ \\
\hline & Multi-gravid & 662 & 387 & & & \\
\hline \multirow[t]{2}{*}{ Occupation of women } & Others & 35 & 2 & $13.21[3.09-79.63]$ & $10.6[2.3-48.34]$ & 0.02 \\
\hline & House wife & 966 & 729 & & & \\
\hline
\end{tabular}

PMTCT service utilization in urban area: Utilization of PMTCT services among ANC attendants in urban area was $68.1 \%$ [95 \% CI: 65 $\%-71 \%]$. Utilization of PMTCT service in urban area was affected by the presence of internal referral system in the health facility, knowledge of PMTCT, gravidity and involvement in PMTCT service (Table 4).

Table 4: Determinants of PMTCT service utilization in urban area $(n=883)$.

\begin{tabular}{|c|c|c|c|c|c|c|}
\hline \multirow[t]{2}{*}{ Variable } & & \multicolumn{2}{|c|}{ Utilization } & \multirow[t]{2}{*}{ COR $[95 \% \mathrm{CI}]$} & \multirow[t]{2}{*}{ AOR $[95 \% C I]$} & \multirow[t]{2}{*}{ p-value } \\
\hline & & Yes & No & & & \\
\hline \multirow{2}{*}{$\begin{array}{l}\text { Internal referral } \\
\text { system }\end{array}$} & Available & 562 & 245 & $0.86[0.56-1.31]$ & $2.12[1.27-3.56]$ & $<0.01$ \\
\hline & Not & 99 & 37 & & & \\
\hline \multirow{2}{*}{$\begin{array}{l}\text { Knowledge on } \\
\text { PMTCT }\end{array}$} & Knowledgeable & 462 & 168 & 2.26 [1.64-3.09] & $2.15[1.51-3.06]$ & $<0.01$ \\
\hline & Not Knowledgeable & 139 & 114 & & & \\
\hline \multirow[t]{2}{*}{ Gravidity } & Primi-gravid & 191 & 152 & $0.4[0.29-0.54]$ & $0.52[0.38-0.72]$ & $<0.01$ \\
\hline & Multi-gravid & 410 & 130 & & & \\
\hline Involvement in & Involved & 71 & 17 & $2.09[1.17-3.76]$ & $2.76[1.49-5.13]$ & $<0.01$ \\
\hline PMTCT service & Not involved & 530 & 265 & & & \\
\hline
\end{tabular}

\section{DISCUSSION}

Utilization of PMTCT service among pregnant women attending ANC was $61.3 \%$ [95\% CI: 59\% - $63 \%$. A significant proportion of pregnant women $(38.7 \%)$ attending ANC in the health facility were missed for PMTCT service. This finding was higher than the Ethiopian demographic and health surveillance report (EDHS) of 2016(22). This is becuase this research

DOI: http://dx.doi.org/10.4314/ejhs.v28i6.13 
is a health facility-based study where the denominators are ANC attendants whereas the EDHS 2011 report was a community-based study where the denominator was all pregnant women in the community.

Only $30.11 \%$ of the pregnant women disclosed their result to their partner. This indicates that pregnant women were not freely discussing the issue of HIV to their partner. The odds of PMTCT service utilization among PMTCT knowledgeable pregnant women were 1.87 times higher than non-knowledgeable pregnant women [AOR 1.87, $95 \%$ CI: 1.53-2.28]. This finding agrees with 2013 world health organization (WHO) report. This is due to the reason that if they know the advantage of PMTCT service, their awareness and willingness to use the service becomes high, Additionally, if they have knowledge on PMTCT they will care for their newborn's health so they try to avoid the risk of mother-to- child transmission by using PMTCT services (23). The odds of PMTCT service utilization in rural women were $33 \%$ higher than the urban women [AOR 0.67, $95 \%$ CI: 0.51$0.89]$. This might be due to the reason that in the urban area women afraid the stigma and discrimination associated with HIV testing in the nearby health facility, so they prefer to take HIV testing outside their catchment areas. Additionally, in the urban area, health professionals suffer from huge workload so they may not implement proper counseling and testing of PMTCT.

The presence of internal referral system inside health facility increases the utilization of PMTCT service by 3.06 folds [AOR 3.06, 95\% CI: $2.51-3.72]$. This finding agrees with the finding from Kenya (15). This is because in the third world countries like Ethiopia, most of pregnant women had low educational status, so they did not know where to go to get the proper service after they receive their test result. Additionally, internal referral system links all tested pregnant women to the next service. Good interaction between pregnant women and health personnel increases the utilization of PMTCT service by 2.18 folds [AOR 2.18, 95\% CI: 1.75 2.76]. This finding agrees with findings from
Rwanda and Uganda $(13,24)$. This is because good interaction increases the level of trust between health personnel and pregnant women (18). Fear about the impact of HIV/AIDS increases the utilization of PMTCT service by 1.23 folds higher [AOR 1.23, 95\% CI: 1.03-1.47]. This is because mothers care about the health of their newborn. They did what they can to get their child free from HIV. Long waiting hour decreases the utilization of PMTCT service by $26 \%$ lower [AOR 0.74, 95\% CI: 0.58-0.94]. This finding was in line with the findings from Nazareth in Ethiopia and a 2013 WHO report $(16,18)$. This is because long waiting hour makes the pregnant women dislike the health facility services and create suboptimal health professionals client interaction. The number of ANC visit already accomplished significantly affects the utilization of PMTCT service, pregnant women that had more than 1 ANC visit already accomplished were 1.79 times more likely to use PMTCT services than women that had only one ANC visit [AOR 1.79, 95\% CI: 1.4-2.29]. This finding agrees with findings from Burkina Faso and Rwanda $(11,12)$. This is because the fact that as the number of ANC visit increases, clients were more likely to get compressive HIV education and more likely to care about the health of their child and themselves (15).

The odds of PMTCT service utilization in multi-gravid women was $45 \%$ higher than primigravid women [AOR 0.55, 95\% CI: 0.46-0.66]. This is because of the fact that those multi-gravid women had exposed themselves to repeated number of counseling and testing for HIV/ AIDS for their previous pregnancy so they are more aware of PMTCT services advantages. Women's education benefits a lot for PMTCT service utilization, the odds of PMTCT service utilization among women with educational status of diploma and above was 1.43 times higher [AOR 1.43, 95\% CI: 1.04-1.96]. This finding agrees with findings from India, Rwanda, and Uganda $(9,12,24)$. This is because educational status increases the adherence of women to health facility services; their knowledge regarding PMTCT will increase as their educational status increases. Involving women in PMTCT services increases the 
utilization of PMTCT services by 2.57 folds higher [AOR 2.57, 95\% CI: 1.51 - 4.4]. This finding agrees with findings from Nazareth and Mekele, Ethiopia $(16,17)$. This is because the community's awareness about PMTCT service will be enhanced by involving them in the planning, implementing and evaluating the PMTCT service program.

The significant proportion of pregnant women attending ANC was missed for PMTCT service utilization. Good knowledge regarding PMTCT, the presence of internal referral system, good health professional-to-client interaction, fear and ANC visit more than one increase the PMTCT service utilization. Urban residents, long waiting hour and primi-gravidity negatively affect PMTCT service utilization.

Every health facility should have an internal referral system. Any PMTCT intervention should involve the community in its planning, implementation and evaluation stage. Emphasis should be given to the urban area health facilities about PMTCT service implementation.

\section{ACKNOWLEDGEMENTS}

We would like to acknowledge the University of Bahir Dar College of Medicine and Health Science for financially sponsoring this research. We would also like to acknowledge the Amhara Regional State Health Bureau for giving the necessary information.

\section{REFERENCES}

Tesfaye N, Ketema T, Halilemariam M, Abebaw Z, Debele B, Mekonene B: National Compresnive PMTCT/MNCH Training Package Refernce Manual Health EFMo. In. Edited by FMOH. Addis Ababa; 2011: 289.

2. UNAIDS: Global plan to wards the elimination of new HIV infections among children by and keeping their mothers alive. In. Edited by UNAIDS. Geneva: UNAIDS; 2009.

3. FHAPCO: Guideline for prevention of mother to child transmission of HIV in Ethiopia. In. Edited by Health FMo. Addis Ababa, Ethiopia; july 2007.
4. HAPCO: Federal HIV/AIDS Prevention and Control Office:In Multi-sectoral HIV/AIDS Response Annual Monitoring and Evaluation Report 2002EFY. In. Addis Ababa: HAPCO; 2009.

5. HAPCO: Federal HIV/AIDS Preventionand Control Office: Multi-sectoralHIV/AIDS Response Annual Monitoring and Evaluation Report 2001 EFY. In. Addis Ababa: HAPCO; 2009.

6. Human Immunideficincy Virus/Aqiured Immunodeficincy Syndrome in Ethiopia [http://en.wikipedia.org/wiki/HIV/AIDS_in Ethiopia]

7. Abelti G, Ahmed J, Seyuom E, Mengistu G, Bogale T, Tamene W. Ethiopia Demographic and Health Survey 2011 In. Addis Ababa: Ethiopia Federal Democratic Republic of Ethiopia Population Census Comission; 2011.

8. Janet T, Laura N. HIV-related Stigma as a Barrier to Achievemen to Global PMTCT and Maternal Health Goals: A Review of the Evidence. AIDS Behav, 2013;17:2528-2539.

9. Mayuri P, Shrinivas D, Vinay K, Sanjeevani K, Ritu P. Socio-demographic factors associated with loss to follow-up of HIVinfected women attending aprivate sector PMTCT program in Maharashtra, India. AIDSCare, 2011; 23(5):593-600.

10. Deschamps M. Overcoming Barriers to Increasing access to PMTCT. In: Meeting of the Expert Panel on the Prevention of Mother-To-Child Transmission. Washington DC USA: The GHESKIO Centers; 2009: 19.

11. Sarker M, Sanou A, Snow R, Ganame J, Gondos A. Determinants of HIV counselling and testing participation in a Prevention of Mother-to-Child Transmission programme in rural Burkina Faso. Tropical Medicine and International Health 2007, 12(1):1475-1483.

12. Delvaux T, Elul B, Ndagije F, Munyana E, Roberfroi D, Asiimwe A. Determinants of Nonadherence to a Single-Dose Nevirapine Regimen for the Prevention of Mother-toChildHIVTransmission in Rwanda journal of aqiured immuno deficinecy syndrome, 2009; 50(2):223-230. 
13. Kebaabetswe P. Barriers to participation in the prevention of mother- to-child HIV transmission program in Gaborone, Botswana a qualitative approach. AIDS Care, 2007;19(3):355-360.

14. Bajunirwe F, Muzoora M. Barriers to the implementation of programmes for the prevention of mother-to-child transmission of HIV: a cross-sectional survey in rural and urban Uganda. AIDS Res Ther, 2005; 2:10.

15. Phelgona O, Pamela K, Rose B, Elizabeth B, Steven M, Grace S. Determinants of failure to access care in mothers referred to HIV treatment programs in Nairobi, Kenya. AIDS Care: Psychological and Socio-medical Aspects of AIDS/HIV, 2010; 22(6):7.

16. Tesfahun H. Determining Factors that Affect Pregnant Women's Utilization of PMTCT Services in Ethiopia: The Case of Health Facilities at Nazareth/Adama Addis Ababa, Ethiopia: Addis Ababa; 2006.

17. Fisaha $H$, Yemane B. Male partner involvements in PMTCT : a cross sectional study, Mekelle, Northern Ethiopia. BMC Pregnancy and Child birth 2014;14:65).

18. Laura F: Women's experiences in services for preventing the mother-to-child transmission of HIV. In. USA: World Health Organization; 2013.

19. Tilahun N, Yoseph W. Analysis of the Prevention of Mother-to-Child Transmission(PMTCT) Service utilization in Ethiopia:2006-2010. Reproductive Health Matters, 2011;8(6):1-8.

20. Lehman D, Stewart J, Overbaugh J. Antiretroviral strategies to prevent mother-tochild transmission of HIV:srtiking a balance between efficacy, feasibility, and resistance. PLoSMed, 2009;6(10): e1000169.

21. Mofenson L. Protecting the next generation-eliminating perinatal HIV1infection. NEnglJMed, 2010;362(24):23162318.

22. Central Statistical Agency - CSA/Ethiopia, ICF. Ethiopia Demographic and Health Sruvey 2016. In. Addis Ababa, Ethiopia: CSA and ICF; 2017.
23. Asefa A, Beyene H. Awareness and knowledge on timing of mother-to-child transmission of HIV among antenatal care attending women in Southern Ethiopia: a cross sectional study. Reproductive Health, 2013, 10(66):1-8.

24. Bajunirwe F, Muzoora M. Barriers to the implementation of programs for the prevention of mother-to-child transmission of HIV:across sectional survey in rural and urban Uganda. AIDS Research Therapy, 2005;2(10):1-7. 